\title{
Treating head and neck tumors during the SARS-CoV-2 epidemic, 2019-2020: Sichuan Cancer Hospital
}

\author{
Yong-Cong Cai, et al. ${ }^{1}$ \\ ${ }^{1}$ Affiliation not available
}

April 28, 2020

\begin{abstract}
Since December 2019, a number of patients with novel coronavirus pneumonia (NCP) have been identified in Wuhan, Hubei Province, China. NCP has rapidly spread to other provinces and cities in China and other countries in the world. Due to the rapid increase in reported cases in China and around the world, on January 30, 2020, the World Health Organization (WHO) Emergency Committee announced that NCP is a Public Health Emergency of International Concern (PHEIC). However, there are relatively few suggestions and measures for tumor patients, especially patients with head and neck tumors. This article summarizes the prevention and control of disease in our medical institution to provide a reference for front-line head and neck surgeons.
\end{abstract}

\section{Full author list}

Yong-Cong Cai, MD¹, Wei Wang, MD ${ }^{1}$, Chao Li, MD, PHD ${ }^{1}$, Din-fen Zeng ${ }^{1}$, Yu-Qiu Zhou, MD ${ }^{1}$, Rong-Hao Sun, MD ${ }^{1}$, Hua Jiang ${ }^{1}$, Hui Guo ${ }^{2}$, Shao-Xin Wang ${ }^{1}$, Jian Jiang, MD ${ }^{1}$

1. Department of Head and Neck Surgery, Sichuan Cancer Hospital \& Institute, Sichuan Cancer Center, School of Medicine, University of Electronic Science and Technology of China, Chengdu, 610041, China.

\section{Introduction}

Since December 2019, a number of patients with novel coronavirus pneumonia (International name, 2019 novel coronavirus disease (COVID-19); China's domestic name, novel coronavirus pneumonia (NCP)) have been identified in Wuhan, Hubei Province, China [1, 2]. With the spread of the epidemic, patients with NCP have been reported in many cities in China as well as in many countries and regions around the world, such as South Korea, Australia, the United States and Italy [3-6]. Currently, the main source of infection is severe acute respiratory syndrome coronavirus 2 (SARS-CoV-2)-infected patients. People with asymptomatic infection may also become a source of infection. Airborne droplets and contact are the main routes of transmission [7]. As of 9 am on March 25, 2020, there were 81850 confirmed cases in China and 3287 cumulative deaths; there were 339081 confirmed cases and 15487 cumulative deaths in other countries.

One study summarized and analyzed the proportion of patients with NCP with tumors and the prognosis of these patients, presenting the first published clinical evidence of the risk level of patients with tumors in the NCP epidemic [8]. Because of reduced immune function, patients with tumors, especially those associated with chronic underlying diseases, are susceptible populations for SARS-CoV-2 infection. For patients with head and neck tumors, particularly those with laryngeal cancer, hypopharyngeal cancer and thyroid tumors with dyspnea, prompt surgical treatment or tracheotomy is needed. For these patients with head and neck tumors, during the NCP epidemic, developing a treatment plan is very important. The National Health Commission of the People's Republic of China has also recently issued guidelines for the diagnosis and treatment of this disease, and these guidelines have been revised several times in the short time since the 
outbreak began, with the latest being the sixth edition. However, there are relatively few suggestions and measures for patients with tumors, especially those with head and neck tumors. This article summarizes the prevention and control of disease in our medical institution to provide a reference for front-line head and neck surgeons.

\section{The situation of tumor patients during the epidemic:}

During the epidemic, patients with tumors have represented a very special patient group. A study that specifically targeted 1,590 patients with $\mathrm{NCP}$ with previous medical records in 575 hospitals found that there were 18 patients with a history of cancer. Compared with NCP patients without cancer, NCP patients with a history of cancer were older (63.1 vs. 48.7 years), and a higher proportion of NCP patients with cancer had a history of smoking (22.2\% vs. $6.8 \%)$, shortness of breath $(47.1 \%$ vs. $23.5 \%)$ or severe symptoms according to computed tomography (CT) imaging (94.4\% vs. $70.8 \%)$. More importantly, the study found that patients with tumors had a higher risk of developing severe disease than did non-tumor patients. Moreover, for all comorbidities, patients with a cancer history had the highest risk of severe disease. In addition, researchers also found that tumor patients with NCP worsened faster than did non-tumor patients (median time from onset to severe symptoms was 13 days vs 43 days, $\mathrm{P}<0.0001)$. Therefore, the research team proposed three strategies for patients with tumors during the NCP outbreak and other possible epidemics of severe infectious diseases in the future: first, in endemic areas, for clinically stable patients with tumors, delayed chemotherapy or elective surgery should be considered; second, patients with tumors and cancer survivors should strengthen their personal protection; and third, if patients with tumors are infected with SARS-CoV2 , especially elderly patients or patients with other comorbidities, extra monitoring or treatment should be provided for priority treatment [8].

\section{Organizational guarantee}

After the outbreak of NCP, our hospital attached great importance to and implemented the corresponding prevention and control measures for patients with head and neck tumors according to the characteristics of these patients. The Department of Head and Neck Surgery quickly set up an Emergency Incident Command Team (EICT). The members include the department director, head nurse, medical and nursing team leaders, infectious disease doctors, infectious disease nurse, and protective material management nurse. The role of this group is to scientifically analyze departmental epidemic situations, reasonably integrate existing resources, develop appropriate infection management procedures and systems that conform to the characteristics of this department, and complete the rational allocation of personnel and materials to fully control the dynamic situation in the department regarding epidemic prevention and control. All staff was required to study the latest diagnostic, treatment and preventive measures for NCP every week.

\section{Innovation in clinical procedures}

The primary measures for prevention and control during this epidemic are to reduce the population flow and close contact among personnel, effectively cut off the infection transmission routes, avoid the occurrence of new NCP cases, and allow prompt and effective quarantine and treatment for people with suspected and confirmed infection. However, due to the establishment of fever clinics or the admission and treatment of suspected cases, hospitals are also the hardest hit areas by cross-infection. For general hospitals, some nonfatal chronic diseases can be handled by reducing or stopping outpatient and inpatient treatment. General hospitals should focus on treating patients with critical severe diseases. For specialized cancer hospitals, the annual death toll due to cancer is much higher than the annual death toll from all currently known infectious diseases, and tumor patients with immune dysfunction are more prone to concurrent infection during the epidemic, leading to fatal complications. Therefore, according to the characteristics of our specialized cancer hospital and the epidemic situation, we set up a three-level triage system in the clinics, changed the layout and environment of the existing department structure, and carried out path-based screening for ward $\operatorname{admission(Fig1).~}$ 


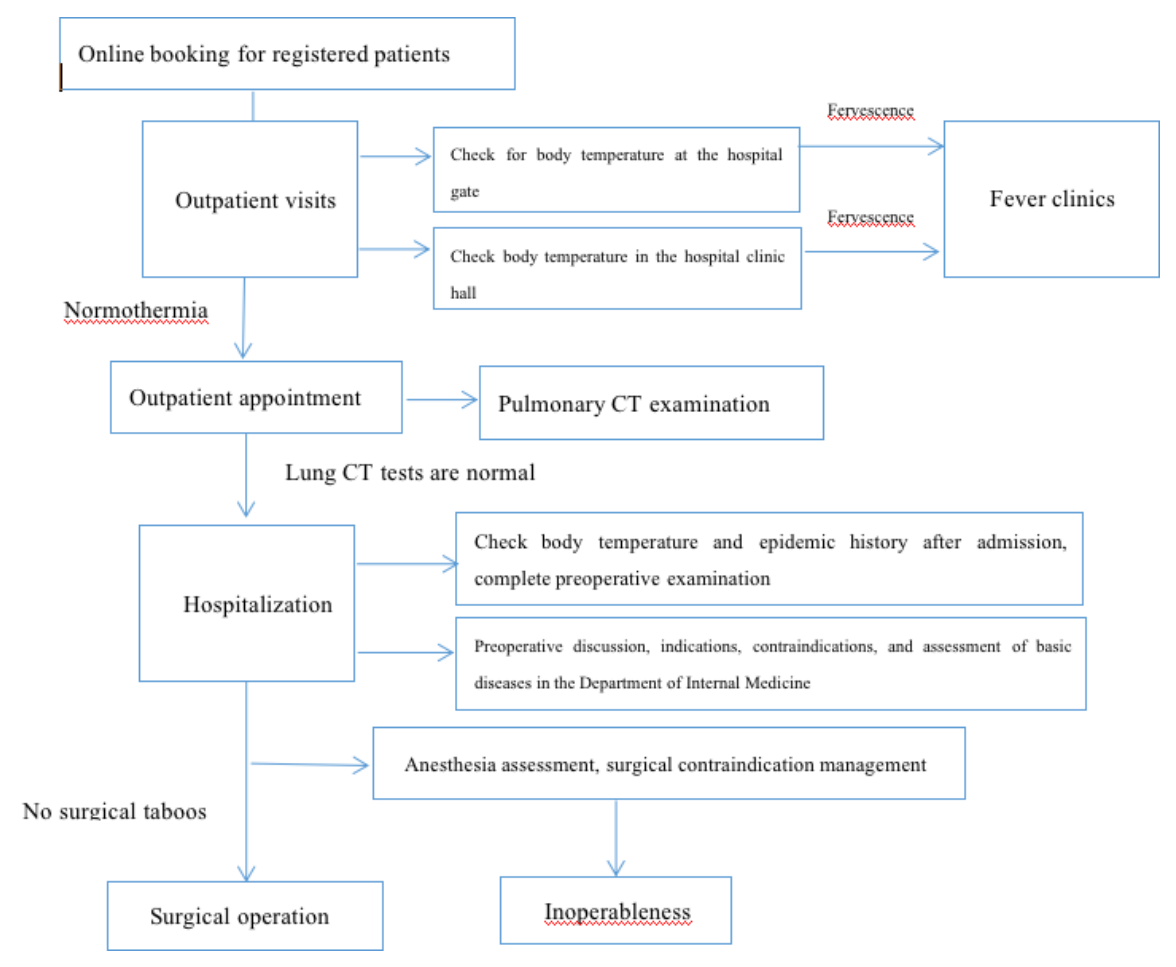

3.1 Establishing a triage area in the fever clinic for tumor patients Our hospitals has effectively separated tumor patients with suspected NCP from tumor patients with fever of unknown origin, promoted the use of masks by anyone visiting the hospital and enhanced community respiratory protection awareness through WeChat, official accounts, official websites and other online media.

\subsection{Implementing the three-level triage system in the emergency department and clinics}

First-level triage : The clinics and emergency department were in charge of triage, which was completely separated from the general counseling station. The essential materials (surgical masks, handheld thermometers, hand sanitizer, etc.) were prepared at the triage sites, and the basic information for the triaged patients (basic information, epidemiological history) was recorded. The epidemiological history, such as the travel history to the epidemic areas or close contact with patients with suspected NCP, was inquired, and the body temperature was measured. Once patients with fever or patients with an epidemiological history were identified, patients and any accompanying persons were immediately given and guided to wear surgical masks. The patient was transferred to the fever clinic by the triage nurse.

Second-level triage : When treating patients, the medical personnel in each diagnostic area of the clinics re-checked the patients and their families regarding their epidemiological history, such as travel history to epidemic areas or close contact with patients with suspected NCP, and recorded vital signs, such as body temperature, pulse, respiration, etc.

Third-level triage : The clinic and emergency physicians asked patients detailed questions regarding fever and cough and obtained further epidemiological history information and asked the patients to sign the "Integrity Commitment regarding the Epidemiological History with Novel Coronavirus Pneumonia".

3.3 Actively control the frequency of patient visits and reduce population aggregation and population flow The risks and recommendations for the diagnosis and treatment of patients with head and neck tumors during the prevention and control of the NCP epidemic were announced through multiple channels, such as the hospital's official account and official website and various WeChat groups for continuing nursing care. An appointment system was implemented to limit outpatient numbers and reduce outpatient 
visits. Additionally, online specialist consultations were set up, and an online platform was used to establish an integrative medical and healthcare team for online consultation.

\section{Reformation of the layout of the department structure}

During the NCP outbreak, the Department of Head and Neck Surgery implemented closed-loop one-way entry and exit management on the basis of existing infrastructure on the ward: entrances and exits signs were clearly set up in the elevator hall entering the ward, and dedicated personnel maintained order and conducted personnel registration and screening. Only one patient was admitted to each room, with up to one accompanying person, and visitations were declined. Medical staff during hospitalization were relatively fixed, and medical personnel and patients were fully protected. Three quarantine wards were set up to facilitate the in situ quarantine of suspected patients. A suspected patient quarantine ward was set up in the inpatient unit in a relatively independent space, which was a negative pressure ward.

\section{Principles of hospitalization treatment}

Patients received in-hospital treatment according to the following principles: emergency and severe patients need to be treated first, and non-emergency and mild patients can be treated later, taking into account complications and underlying diseases; elective surgeries need to be postponed; and the treating doctor needs to be contacted if a patient's condition changes during routine follow-up. The speed and quantity of admission were controlled to ensure the safety of patients and medical personnel during the epidemic. For patients with benign head and neck tumors or slow-progressing malignant tumors, such as low-risk differentiated thyroid cancer, because the tumor will not progress rapidly in the short term and cause serious complications, the in-hospital treatment of such patients has been postponed until the epidemic stabilizes.

\section{Preparation for admission and screening}

After the outpatient doctor completed the third-level triage and issued the admission orders, an appointment was scheduled. In addition, a network communication platform was established with the patients or their families, and the network communication lists for patients admitted to the Department of Head and Neck were distributed to the patients and their families. Network screening of the preadmission epidemic situation was performed. Additionally, a complete blood count was conducted, and chest CT examinations were performed if necessary. At the time of admission, each patient's body temperature was checked at the entrance of the surgical building. If the body temperature was normal, patients were admitted to the head and neck surgical unit. Body temperature was screened again at the entrance of the unit by the nurses, who used secondary protective measures. Body temperature was measured with an electronic thermometer, the close contact history with NCP patients was obtained, the patient and any accompanying persons signed the "Integrity Commitment regarding Patient and Family Epidemiological History with Novel Coronavirus Pneumonia", and the patient and any accompanying persons completes the sign-in sheets. If there were no abnormalities, patients went to the nurse station to complete the admission procedure. In addition to regular basic and specialist assessments, the patients were mainly assessed for respiratory function and past respiratory disease history.

\section{Preoperative examination}

The doctor in charge prescribed blood tests and chest CT, as they are mandatory examinations for the NCP epidemic. The staff made a joint appointment and provided the date to the patient. The patient entered the unit and the surgical building with an examination reservation sheet and a wristband. Nurses assessed patient self-care and managerial abilities. If there were no abnormalities, under normal circumstances, patients could only be accompanied by the accompanying person when agreement for the endoscopic examination had to be signed by a family member, and the accompanying person wore an accompanying badge and wristband. The purpose of these measure was to control population flow in the medical technology department area and reduce the number of cross-infections.

\section{Surgical patients}


Operations were performed only when normal results according to evaluations by the Head and Neck Surgery Center, Internal Medicine Center and Anesthesiology Center could be achieved. The patient was admitted to the operating room under the following conditions: with a chest CT scan within the prior 7 days, having signed the "Integrity Commitment regarding the Epidemiological History with Novel Coronavirus Pneumonia", wearing a mask, and having a surgical information sheet indicating vital signs. Postoperative vital signs were closely monitored, and the risks of exposure to NCP were strictly assessed. Patients were encouraged to get out of bed as soon as possible after surgery, with precise nutrition management.

\section{Postoperative multidimensional integrative medical care at home}

During the NCP epidemic, tumor patients at home still have different degrees of risks of immune dysfunction and susceptibility to infection during the postoperative rehabilitation period. We not only pay attention to the prevention and control of virus infection of inpatients and the NCP epidemic in the hospital but also provide at-home psychological support and care related to NCP to patients with tumors. Through a nursing WeChat platform and a third-party online platform, we implemented integrative medical care at home and sent notifications regarding information about protection against NCP for patients with tumors.

\section{Reexamination and follow-up of patients with head and neck tumors}

For the follow-up of patients during the NCP epidemic, online follow-up or follow-up by telephone is preferred to reduce population flow and cross-infection. For patients with differentiated thyroid cancer and other diseases with relatively slow progression and patients recovered at the 1-year follow-up, the follow-up time was extended to 1-3 months. For patients who must complete follow-up, neighborhood community hospitals are recommended for the necessary examinations, and guidance is provided after the examination results are provided online. During follow-up, medical staff is in charge of both anticancer and antiepidemic responsibilities. In addition to inquiring about cancer-related information, the medical staff must also provide health guidance related to epidemic prevention.

From February 1 to March 10,2020, during the epidemic of new coronavirus infection, our head and neck surgery center completed 97 operations, including 86 for thyroid cancer, 5 for oral cancer (4 for free flap reconstruction and 4 for tracheotomy),2 for parotid gland , 1 for squamous cell carcinoma of the scalp ,2 for cervical lymph node biopsy and 1 for laryngeal cancer. Seven patients had postoperative fever, the highest body temperature was $39^{\circ}$, and all patients returned to normal body temperature on the third day after operation; A case of thyroid cancer patients with fever on the first day after operation with cough symptoms, the lung CT did not rule out a new type of coronavirus infection, so a new type of coronavirus test, two consecutive tests were negative, the patient's body temperature returned to normal on the third day after operation.All patients were followed up 2 weeks after operation, without fever symptoms, and all doctors and nurses had no fever symptoms.

\section{Summary}

It is very rare for head and neck surgery departments in cancer hospitals to face sudden epidemic situations, such as the NCP epidemic. There are relatively few clinical prevention and control measures and nursing experience, which causes enormous challenges regarding diagnosis, treatment and nursing in the Department of Head and Neck Surgery. Individualized diagnosis and treatment and care should be carried out according to the characteristics of the tumor. For examinations, treatment and nursing care involving the mouth, care should be taken regarding the personal protection of medical personnel. Therefore, under the premise of raising awareness of epidemic prevention and control in the whole hospital, the source of infection should be controlled, population aggregation and population flow need to be reduced, disinfection and isolation measures need to be strictly enforced, and the transmission routes need to be effectively cut off, thereby minimizing and eliminating cross-infection among doctors, nurses, and patients. Under the premise of the prevention and control of the epidemic, we also should focus on humanity, performing epidemiological monitoring and postoperative rehabilitation care for patients and their families. We believe that through the efforts of everyone, we will be able to overcome this virus and overcome cancer. 
Conflict Of Interest We have no conflict of interest.

Funding None

\section{References:}

1. Wang, FS; Zhang, C; What to do next to control the 2019-nCoV epidemic?[J].Lancet.2020,395(10222):391393

2. Wang, D; Hu, B; Hu, C; et al.Clinical Characteristics of 138 Hospitalized Patients With 2019 Novel Coronavirus-Infected Pneumonia in Wuhan, China.[J].JAMA.2020,():

3. Kim JY, Choe PG, Oh Y,etal.The First Case of 2019 Novel Coronavirus Pneumonia Imported into Korea from Wuhan, China: Implication for Infection Prevention and Control Measures.J Korean Med Sci. 2020 Feb 10;35(5):e61. doi: 10.3346/jkms.2020.35.e61.

4. 2019-nCoV National Incident Room Surveillance Team.2019 nCoV acute respiratory disease, Australia Epidemiology Report 1 (Reporting week 26 January - 1 February 2020).Commun Dis Intell (2018). 2020 Feb 6;44. doi: 10.33321/cdi.2019.44.13.

5. Michelle L Holshue, Chas DeBolt, Scott Lindquist, et al. First Case of 2019 Novel Coronavirus in the United States. N Engl J Med 2020. PMID 32004427

6.Marta Giovanetti , Domenico Benvenuto, Silvia Angeletti , The First Two Cases of 2019-nCoV in Italy: Where They Come From? J Med Virol 2020. PMID 32022275

7. Lu, CW; Liu, XF; Jia, ZF; 2019-nCoV transmission through the ocular surface must not be ignored.[J].Lancet.2020,():

8. Wenhua Liang, Weijie Guan, Ruchong Chen, et al. Tumor patients in SARS-CoV-2 infection: a nationwide analysis in China. Lancet Oncol,2020.Feb14,DOI:https://doi .org/10.1016/S1470-2045(20)30096-6. 